\title{
Long non-coding RNA SOX21-AS1 promotes cell proliferation and invasion through upregulating PAK7 expression by sponging miR-144-3p in glioma cells
}

\author{
S. Y. GAI ${ }^{1}$, Z. H. YUAN ${ }^{2, *}$ \\ ${ }^{1}$ Department of Neurosurgery, The Fourth Affiliated Hospital Zhejiang University School of Medicine, Jinhua, Zhejiang, China; ${ }^{2}$ Department of \\ Neurosurgery, The Second Affiliated Hospital of Xian Medical University, Xian, Shaanxi, 710038, China \\ ${ }^{\star}$ Correspondence: ZhihaiYuandfg@163.com
}

Received May 9, 2019 / Accepted August 11, 2019

\begin{abstract}
In this study, the function of long non-coding RNA SOX21 antisense RNA 1 (SOX21-AS1) in the progress of glioma was explored. RNA and protein levels were measured via quantitative reverse transcription-PCR (qRT-PCR) and western blot analysis. In addition, we examined cell proliferation, apoptosis, migration and invasion. The interaction between SOX21AS1 (PAK7) and miR-144-3p was determined via RNA immunoprecipitation (RIP) assay and Luciferase reporter assay. SOX21-AS1 was upregulated in glioma tissues and cells. SOX21-AS1 knockdown was carried out in glioma cells (U251 and U87 cells). Moreover, in vitro, SOX21-AS1 knockdown repressed proliferation, migration, invasion and enhanced apoptosis in glioma cells. In vivo, SOX21-AS1 knockdown suppressed tumor growth in mice. In addition, SOX21-AS1 could sponge miR-144-3p, which was determined to bind to PAK7. miR-144-3p knockdown promoted proliferation, migration, invasion and inhibited cell apoptosis. Importantly, the effects of SOX21-AS1 knockdown-induced proliferation, migration, invasion, and apoptosis were alleviated in glioma cells co-transfected with SOX21-AS1 and miR-144-3p knockdown. Furthermore, miR-144-3p knockdown also attenuated Wnt/ $\beta$-catenin pathway-associated protein levels induced by SOX21-AS1 knockdown. These results indicated that SOX21-AS1/miR-144-3p/PAK7 axis played an oncogenic role in glioma cells by regulating $\mathrm{Wnt} / \beta$-catenin pathway, which suggests a rational therapeutic strategy for glioma.
\end{abstract}

Key words: glioma, SOX21-AS1, miR-144-3p, PAK7, migration, invasion

Glioma is the most common lethal neoplasm of the brain, which accounts for $80 \%$ of brain tumors $[1,2]$. There is increasing incidence worldwide in glioma with 10,000 new diagnoses per year $[3,4]$. Up to date, some therapeutic methods, such as surgical resection, radiotherapy and chemotherapy, have been applied in the treatment of patients with glioma [5-8]. Although surgery, which was used to combine with postoperative chemotherapy and radiotherapy, can improve the patients with glioma to some extent, poor prognosis and high recurrence are still existent [9-11]. Hence, in order to investigate the mechanism underlying curative therapy for glioma, the effective early markers are needed to be explored, which is useful for the diagnosis and prognosis of glioma.

Long noncoding RNAs (lncRNAs), >200 nucleotides, are a class of non-protein-coding RNAs and lack transcriptional ability [12-14]. LncRNAs are involved in cellular development and differentiation, growth, cell cycles, apoptosis in multiple tumors [15-17]. More and more evidence has shown that aberrant lncRNA expression played an important role in human diseases, including tumors [18-21]. Interestingly, lncRNA SOX21 antisense RNA 1 (SOX21-AS1), as a new lncRNA, drew our attention. SOX21-AS1 is located on chromosome 13q32.1 and can be transcribed into a 2986 nucleotide transcript [22]. It is reported that SOX21AS1 could act as an oncogenic molecule in some cancers, including colorectal cancer [23], lung adenocarcinoma [22], oral cancer [24]. However, the mechanism of SOX21-AS1 participation in glioma is still unclear.

LncRNAs have been reported to interact with RNA [25]. This property, at the transcriptional and post-transcriptional level, is beneficial for aiding in the regulatory role [26]. Interestingly, miR-144-3p, which could bind with Frizzled-7 (FZD7), inhibited the progress of glioblastoma [27]. Besides, p21-activated kinase 7 (PAK7), also called PAK5, is a new member of the mammalian PAK family of serine/threonine kinases [28-30]. PAK7 was identified from the mammalian brain and facilitated the induction of filopodia [31]. Recently, accumulating evidence showed that PAK7 was highly expressed in some cancers, such as colon cancer [32], 
ovarian cancer [33], gastric cancer [34], glioma [35, 36], and enhanced cell proliferation, migration and suppressed cell apoptosis. However, whether the miR-144-3p regulates the PAK7 in glioma is unreported.

In the current study, we investigated the role of SOX21AS1 in glioma. In addition, we confirmed that SOX21-AS1/ miR-144-3p/PAK7 axis showed an important role in glioma, which provides a new target for glioma treatment.

\section{Materials and methods}

Tissue collection. Total 40 paired glioma cancer tissues and normal tissues were collected via surgical resection from 2018 to 2019 at The Second Affiliated Hospital of Xi'an Medical University. Among them, there were 13 low-grade gliomas (LGGs) and 30 high-grade gliomas (13 grade III and 14 grade IV tumors). Before surgery, none of the patients received radiotherapy or chemotherapy. Written informed consents were obtained from patients. The experiments were conducted and approved by World Medical Association Declaration of Helsinki and The Second Affiliated Hospital of Xi'an Medical University. The samples were immediately stored in liquid nitrogen until used.

Cell culture and transfection. The human glioma cell lines (U251, U118, LN229, U87 and SHG44), normal human astrocytes (NHA) and HEK293 cells were purchased from ATCC, and they were grown in RPMI-1640 with 10\% fetal bovine serum (FBS) at $37^{\circ} \mathrm{C}$ with $5 \% \mathrm{CO}_{2}$. The U251 and U87 cells were transfected with SOX21-AS1 shRNA or negative control shRNA (shNC), respectively.

Quantitative reverse transcription-PCR (qRT-PCR). Total RNA was extracted from glioma tissues and cells with TRIzol reagent (Invitrogen, Grand Island, NY, USA). Then, cDNA, via a PrimeScript RT reagent Kit (Khayal Bio, Wuhan China), was synthesized using the isolated RNA, and qRT-PCR was conducted. The SOX21-AS1, PAK7 and miR-144-3p levels were determined through SYBR Green (Meilunbio, Dalian, China). GAPDH and U6 were used as controls. The relative expression of SOX21-AS1, PAK7 and miR-144-3p was calculated via $2^{-\Delta \Delta \mathrm{Ct}}$ method. The primers are listed in Table 1.

Table 1. The primers used in this study.

\begin{tabular}{ll}
\hline Name & Sequence (5'-3') \\
\hline SOX21-AS1 & F-GGCTCAGGTTTAGGCGAGTG \\
& R-AGAGGCTCTCCTACTCGTGC \\
PAK7 & F-CCAAAGCCTATGGTGGACCC \\
& R-AGGCCGTTGATGGAGGTTTC \\
GAPDH & F-AGCCACATCGCTCAGACAC \\
& R-GCCCAATACGACCAAATCC \\
miR-144-3p & F- GCCCCTACAGTATAGATGATGTA \\
& R-GTGCAGGGTCCGAGGT \\
U6 & F-CTGGTAGGGTGCTCGCTTCGGCAG \\
& R-CAACTGGTGTCGTGGAGTCGGC \\
\hline
\end{tabular}

Cell viability detection. The cells $\left(2 \times 10^{3}\right.$ cells/well $)$ were seeded into 96 -well plates. Cell viability was measured every $24 \mathrm{~h}$. At $24 \mathrm{~h}, 48 \mathrm{~h}, 72 \mathrm{~h}$ and $96 \mathrm{~h}$, cell counting kit-8 (CCK-8) reagents ( $10 \mu \mathrm{l} /$ well, Dojindo, Tokyo, Japan) were added into wells. After incubation for $4 \mathrm{~h}$ at $37^{\circ} \mathrm{C}$, the optical density (OD) at $450 \mathrm{~nm}$ was read via a microreader.

Flow cytometry analysis. Cells were collected and resuspended in PBS. For cell apoptosis detection, the cells were stained with FITC-labeled Annexin $\mathrm{V}$ as well as propidium iodide (PI) (Beyotime, Shanghai, China) for $15 \mathrm{~min}$. For cell cycle detection, the cells were resuspended in PBS and incubated with PI for $30 \mathrm{~min}$. At last, the cell apoptosis and cell cycle were analyzed using flow cytometry.

Cell migration assay. Cells $\left(1 \times 10^{5}\right.$ cells/well $)$ were seeded into 12 -well plates. At $0 \mathrm{~h}$, wounds were produced with $200 \mu \mathrm{l}$ pipette tip. After washing with PBS, the cells were continually cultured for $24 \mathrm{~h}$. The images were captured at $0 \mathrm{~h}$ and $24 \mathrm{~h}$, and wounded area was calculated.

Transwell invasion assay. Cells $\left(2 \times 10^{4}\right.$ cells/well $)$ were seeded into the upper chambers, which were pre-coated with Matrigel. In addition, complete medium was added into lower chamber. Following incubation for $24 \mathrm{~h}$, the cells were stained with $0.1 \%$ crystal violet (Beyotime, Shanghai, China). The photograph was obtained and invasion cells were counted under a microscope.

Luciferase reporter assay. The sequences of wild-type (WT) SOX21-AS1 containing miR-144-3p binding site or mutant (MUT) SOX21-AS1 losing miR-144-3p binding site were amplified and inserted into pmirGLO vectors. In addition, PAK7 3'-UTR sequences that contain miR-144-3p binding site or lose miR-144-3p binding site were obtained via PCR amplification and cloned into pmirGLO vectors. HEK293 cells were co-transfected with the appropriate luciferase vectors and miR-144-3p mimic or negative control mimic (NC mimic). After $48 \mathrm{~h}$, the luciferase activity was examined through Dual-Luciferase Reporter Gene Assay kit (Promega, Beijing, China).

RNA immunoprecipitation (RIP) assay. SOX21-AS1 was overexpressed in U251 and U87 cells. Afterwards, these cells were subjected to RIP assays using with RNA Immunoprecipitation Kit (SCBIO, Guangzhou, China). In addition, an Ago2 antibody (No. ab32381; Abcam, Shanghai, China) was used. The expression of the SOX21-AS1 and miR-144-3p was detected via qRT-PCR.

Western blot analysis. The proteins from cells were extracted with RIPA. The proteins were determined via BCA protein assay kit (Yubiotech, Shanghai, China), and separated on SDS-PAGE. Then, the samples were transferred onto PVDF membranes (Thermo Fisher Scientific, Shanghai, China). Afterwards, the membranes were blocked with $5 \%$ non-fat milk for $1 \mathrm{~h}$ at $37^{\circ} \mathrm{C}$, incubated with primary antibodies at $4^{\circ} \mathrm{C}$ overnight, and then incubated with secondary antibody (1:1000, Abcam, Cambridge, UK) for $45 \mathrm{~min}$ at $37^{\circ} \mathrm{C}$. Finally, the bands were visualized with ELC (Beyotime, Shanghai, China). Following primary antibodies 
were used: PAK7 (ab110069; 1:500), GSK3 $\beta$ (ab93926; 1:1000), p-GSK3 $\beta$ (ab75745; 1:1000) and $\beta$-catenin (ab6302; 1:1000) (all Abcam, Shanghai, China). Cyclin D1 (No.2922; 1:2000), C-myc (No. 9402; 1:1000), Cleaved caspase 3 (No. 9661; 1:1000), Cleaved caspase 9 (No. 9505; 1:1000), E-cadherin (No. 14472; 1:1000), N-cadherin (No. 4061; 1:1000) and GAPDH (No. 5174; 1:5000) (all Cell Signaling Technology, Danvers, MA, USA).

Tumor growth in nude mice. Six-week-old male nude mice were obtained from Vitalriver Co. (Beijing, China). They were maintained at $12 \mathrm{~h}$ light and dark cycles. All experiments involving mice were conducted in accordance with Guide for the Care and Use of Laboratory Animals and approved by The Second Affiliated Hospital of Xian Medical University. Five million U251 cells stably transfected with SOX21-AS1 shRNA (Lv-shRNA) and control (Lv-ctrl) mediating by lentivirus were subcutaneously injected into nude mice. After that, tumor growth was monitored and detected every week for 5 weeks. The tumor volume $\left(\mathrm{mm}^{3}\right)$ $=$ length $\times$ width $^{2} \times 0.5$. Then, the mice were sacrificed by sodium pentobarbital and tumor tissues were collected. The tissues were photographed and weighed.

Statistical analysis was carried out via SPSS 17.0 software and presented as mean \pm SD. Statistical analysis was carried out using the Student's test, one-way and two-way ANOVA. Kaplan-Meier method was used to estimate recurrence-free survival. Survival rates were calculated at 0-, 20-, 40-, and 60 -month intervals. To determine the relationship between
SOX21-AS1 and miR-144-3p, between SOX21-AS1 and PAK7, a Cox regression model was performed. A p-value $<0.05$ was considered significant. All experiments were repeated 3 times.

\section{Results}

SOX21-AS1 was upregulated in glioma tissues and cells. We conducted qRT-PCR to detect the SOX21-AS1 level. The results showed that the upregulation of SOX21-AS1 was found in glioma tissues compared with normal tissues (Figure 1A). In addition, among low-grade gliomas (I-II), III and IV gliomas, SOX21-AS1 level was highest in grade IV gliomas while was lowest in low-grade gliomas (Figure 1B). Glioma patients with high SOX21-AS1 expression showed shorter survival than glioma patients with low SOX21-AS1 expression (Figure 1C). SOX21-AS1 expression was significantly higher in human glioma cell lines than that in NHA cells (Figure 1D). These results suggested that SOX21-AS1 was upregulated in glioma tissues and cells.

SOX21-AS1 knockdown inhibited glioma cell proliferation and invasion. SOX21-AS1 knockdown was successfully carried out in both U251 and U87 cells, and qRT-PCR showed low SOX21-AS1 expression in glioma cells with SOX21-AS1 knockdown (Figure 2A). CCK-8 assay demonstrated that SOX21-AS1 knockdown repressed cell proliferation in U251 and U87 cells (Figure 2B). Apoptotic cells (Annexin V-FITC) $)^{+} / \mathrm{PI}^{+}$and Annexin V-FITC) ${ }^{+} / \mathrm{PI}^{-}$) were
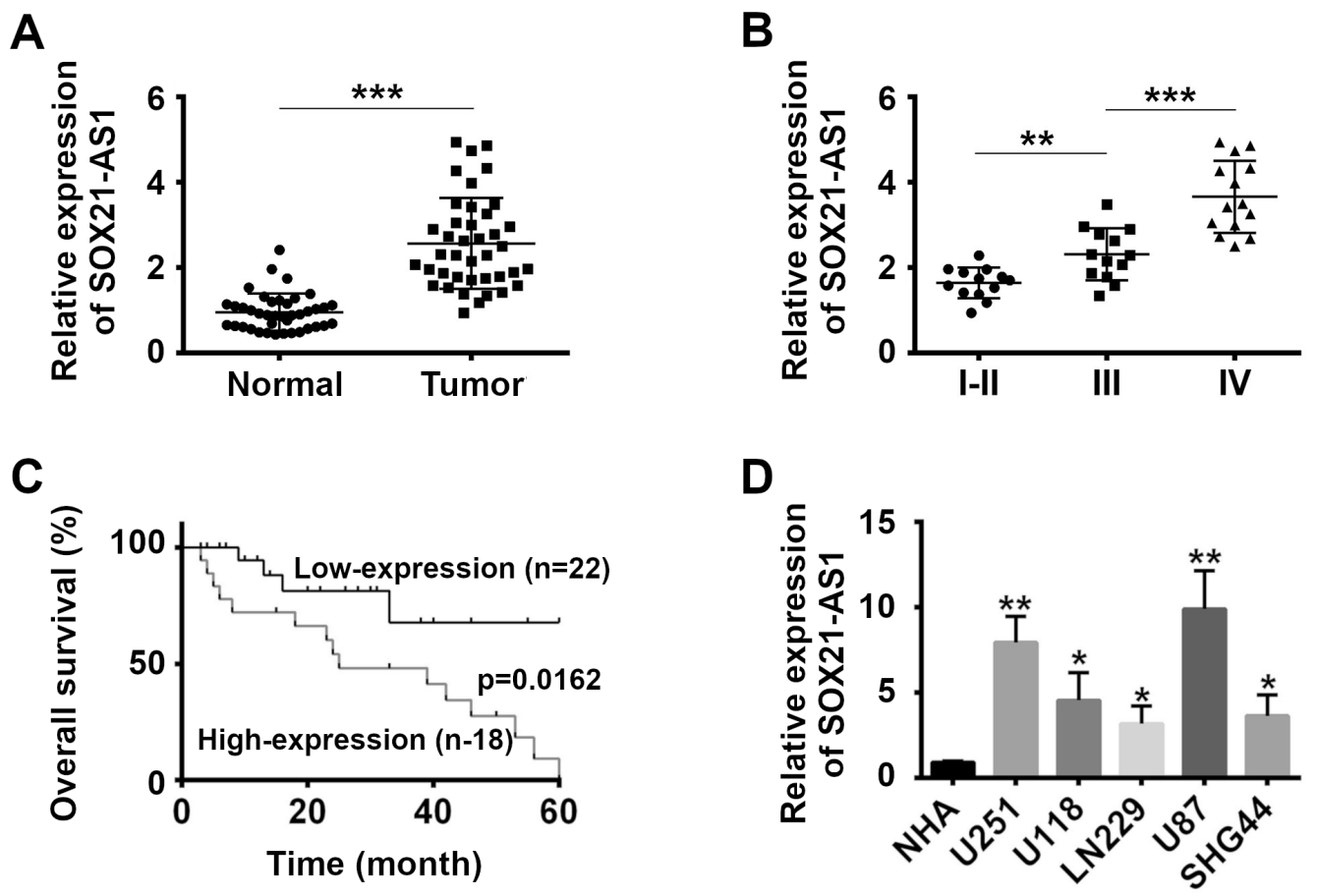

Figure 1. SOX21-AS1 was upregulated in glioma tissues and cells. A, B) SOX21-AS1 level was determined via qRT-PCR in tissues from glioma patients. C) Survival was calculated in glioma patients with SOX21-AS1 expression. D) SOX21-AS1 level was measured through qRT-PCR in glioma cells. Data were presented as mean $\pm \mathrm{SD}$. Statistical analysis was carried out using the student's test and one-way ANOVA by Tukey. ${ }^{*} \mathbf{p}<0.05,{ }^{* *} \mathrm{p}<0.01,{ }^{* * *} \mathrm{p}<0.001$. 

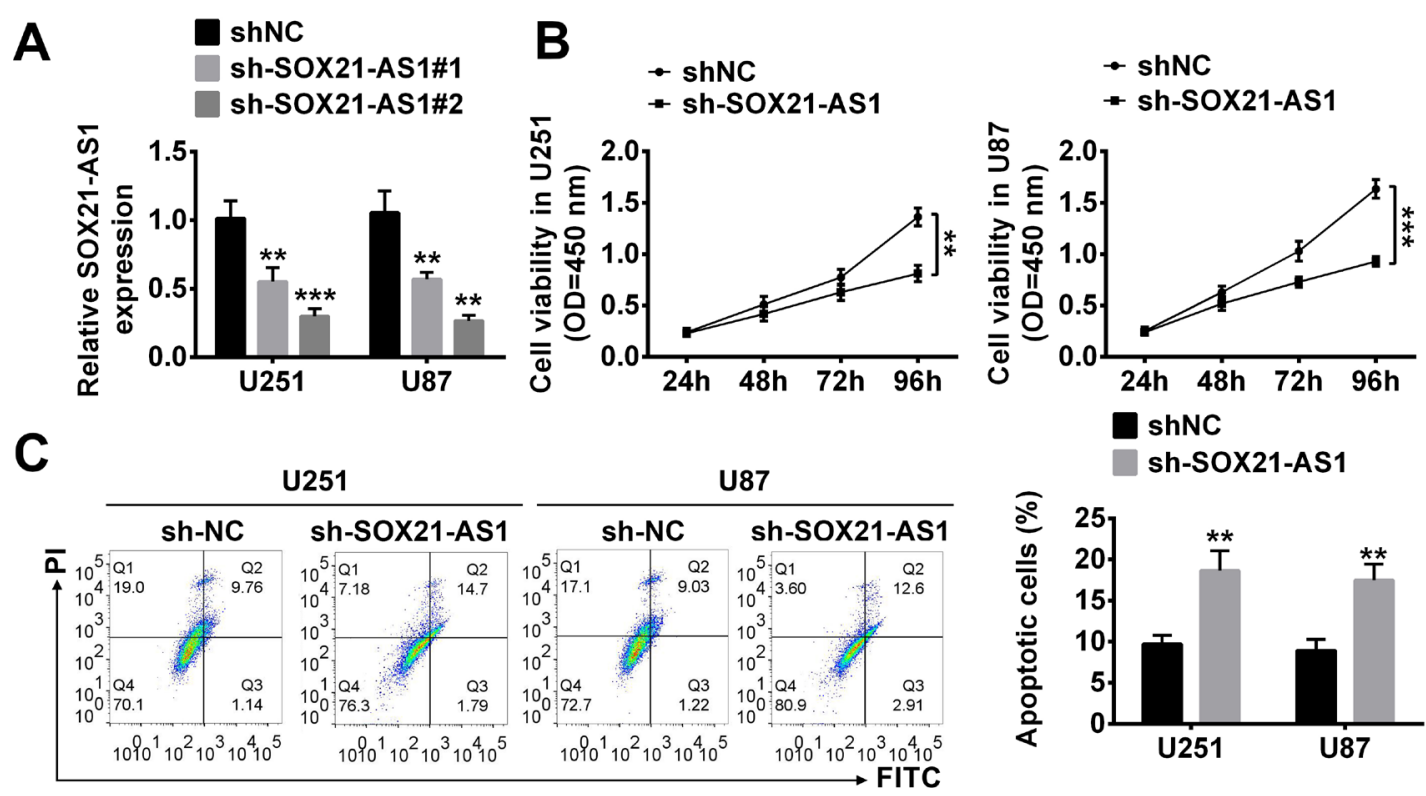

D

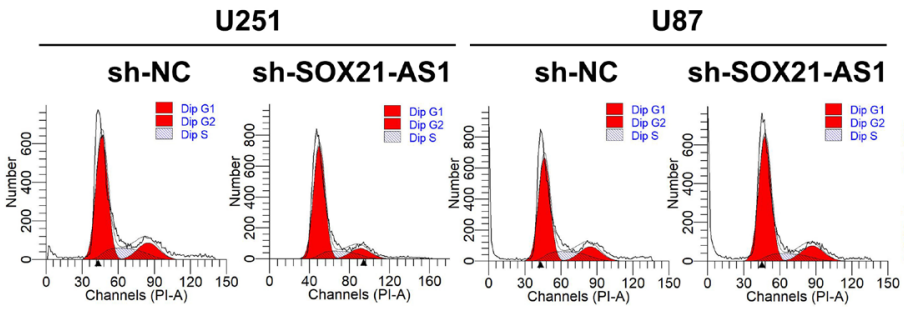

E

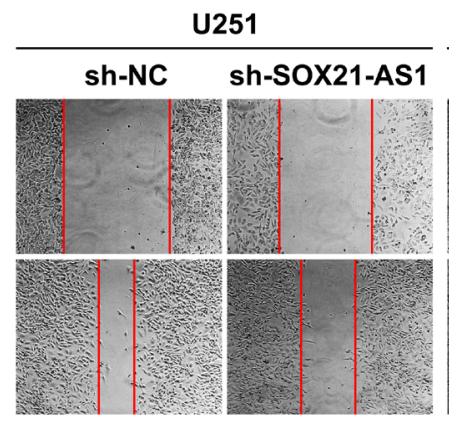

$\mathbf{F}$

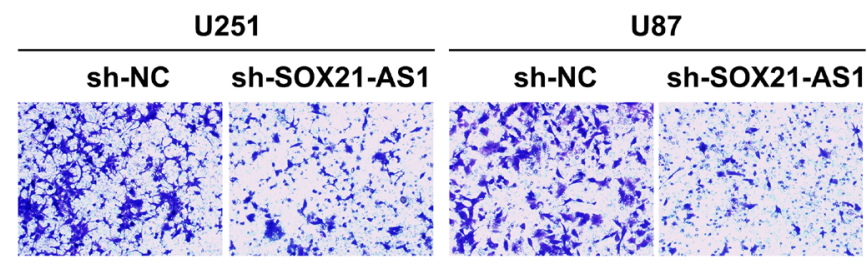

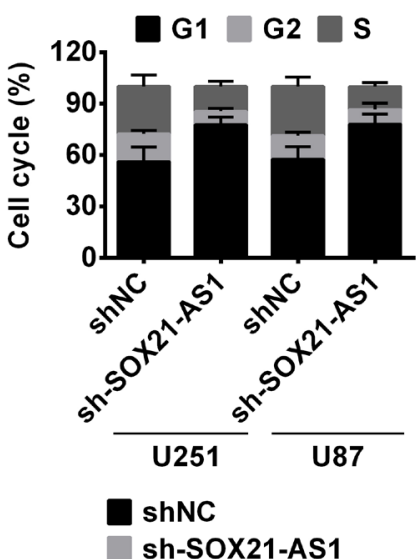

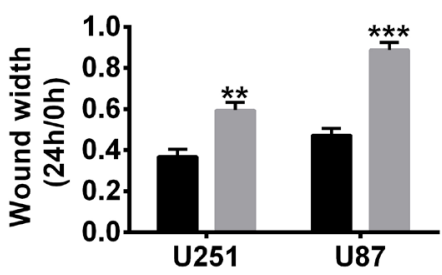

shNC

sh-SOX21-AS1

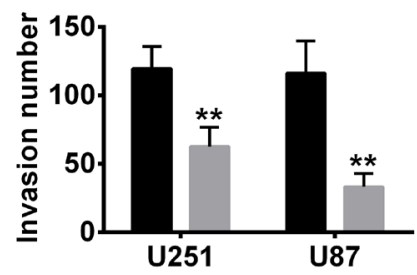

Figure 2. SOX21-AS1 knockdown inhibited glioma cell proliferation and invasion. A) qRT-PCR was used to detect SOX21-AS1 level in U251 and U87 cells. B) CCK-8 assay examined cell proliferation. C and D) Flow cytometry was used to detect cell apoptosis and cell cycle. E and F) Scratch wound healing assay and transwell assay measured the cell migration and invasion. Data were presented as mean \pm SD. Statistical analysis was carried out using the student's test, one-way and two-way ANOVA by Tukey. ${ }^{* *} \mathrm{p}<0.01,{ }^{* * *} \mathrm{p}<0.001$. 
elevated in U251 and U87 cells with SOX21-AS1 knockdown by flow cytometry analysis (Figure 2C). Further cell cycle analysis showed that SOX21-AS1 knockdown increased the cells at G1-stage and decreased cells at S-stage (Figure 2D). In addition, SOX21-AS1 knockdown suppressed cell migration and invasion in both U251 and U87 cells (Figures 2E, 2F). These data indicated that SOX21-AS1 knockdown inhibited glioma cell proliferation and invasion.

SOX21-AS1 knockdown suppressed tumor growth in mice in vivo. The effect of SOX21-AS1 knockdown on tumor growth in mice was evaluated. The qRT-PCR detection revealed that SOX21-AS1 expression was decreased in SOX21-AS1 knockdown tumor tissues (Figure 3A). The inhibition of volume in tumor tissues was induced by SOX21-AS1 knockdown (Figures 3B, 3C). Additionally, SOX21-AS1 knockdown remarkably repressed of the tumor weight (Figure 3D). These findings suggested that SOX21AS1 knockdown suppressed tumor growth in mice in vivo.

SOX21-AS1 was verified to be a target of miR-144-3p. The bioinformatic analysis found that SOX21-AS1 was a target of miR-144-3p (Figure 4A). Luciferase reporter assay showed that miR-144-3p mimics had a suppressive role in SOX21-AS1-WT group, whereas the luciferase activity was not affected in miR-144-3p mimics + SOX21-AS1-MUT group (Figure 4B). RIP assay revealed that the amount of SOX21-AS1 and miR-144-3p immunoprecipitated with
Ago2 was high compared with the respective input group and IgG group, and IgG was significantly lower than SNRNP70 (Figure 4C), suggesting SOX21-AS1 indeed directly binds to miR-144-3p. Then, qRT-PCR determined that SOX21AS1 expression was dramatically elevated in both U251 and U87 cells with SOX21-AS1 overexpression, and SOX21AS1 knockdown promoted miR-144-3p expression while SOX21-AS1 overexpression inhibited miR-144-3p expression (Figure 4D). In addition, miR-144-3p expression was lower in glioma tissues than normal tissues, and miR-144-3p expression showed a lower level in grade III gliomas than that in low-grade gliomas, in grade IV gliomas than grade III gliomas (Figure 4E). miR-144-3p expression was negatively correlated with SOX21-AS1 expression (Figure 4F). These data suggested that SOX21-AS1 was verified to be a target of miR-144-3p.

miR-144-3p targeted PAK7. Targetscan was used to predict that miR-144-3p targeted PAK7 (Figure 5A). Further luciferase reporter assay testified that there was a significant difference between miR-144-3p mimics + PAK7-WT and NC mimics + PAK7-WT. However, there was no effect on PAK7-MUT group (Figure 5B). Moreover, qRT-PCR verified that the increase of miR-144-3p level and decrease of PAK7 level were detected in miR-144-3p mimics U251 and U87 cells, whereas miR-144-3p and PAK7 showed opposite level in miR-144-3p inhibitor U251 and U87 cells (Figure 5C).
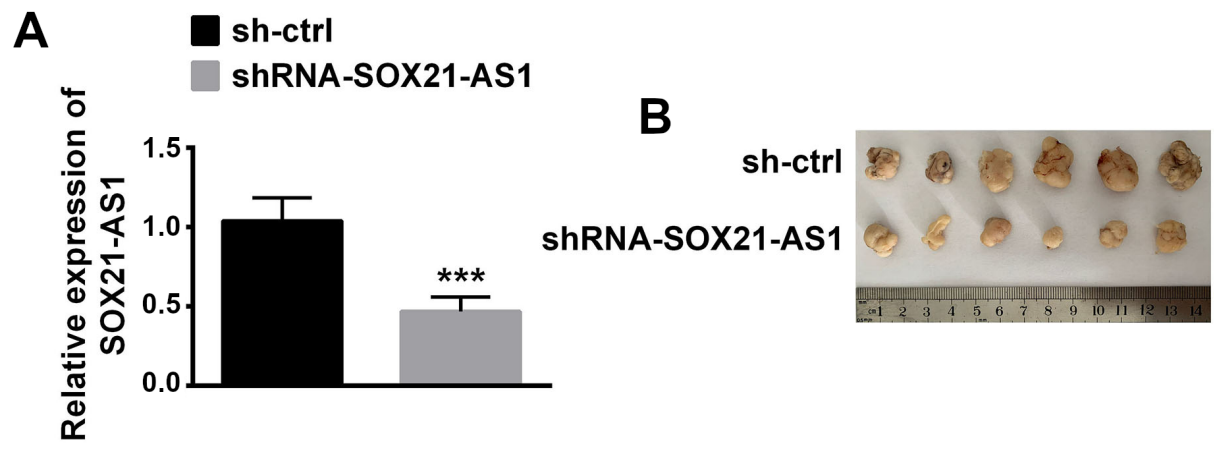
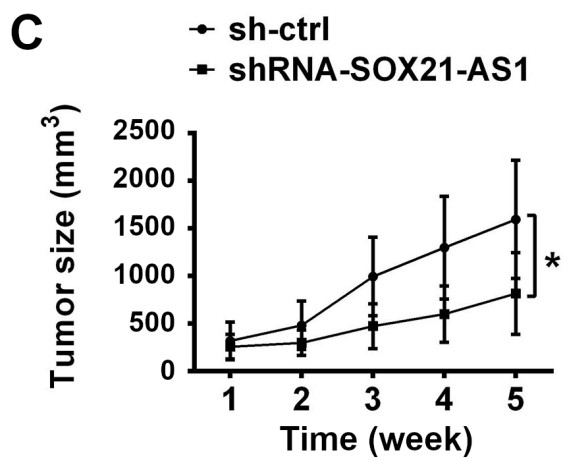

D

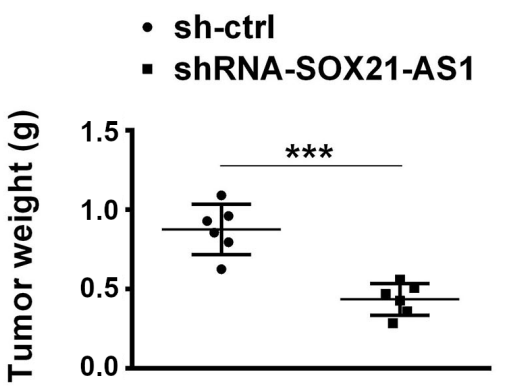

Figure 3. SOX21-AS1 knockdown suppressed tumor growth in mice in vivo. A) SOX21-AS1 level was determined in tissues from mice using qRT-PCR. $\mathrm{B}$ and C) Tissues from mice were photographed and tumor volume was calculated. D) Tumor tissues from mice were weighed. Data were presented as mean \pm SD. Statistical analysis was carried out using the student's test and one-way ANOVA by Tukey. ${ }^{*} \mathrm{p}<0.05,{ }^{\star * *} \mathrm{p}<0.001$. 
A SOX21-AS1 WT '-GGAAUAUUAAAUAUACUGUU-3'
miR-144-3p 3'-UCAUGUAGUAGAUAUGACAU-5'

SOX21-AS1 MUT 5'-GGAAUAUUAAAAUAUGACAU-3'

C

U251

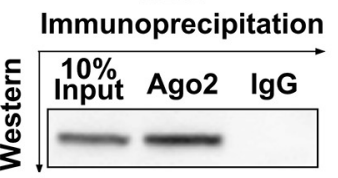

Input

Anti-Ago2

Anti-lgG

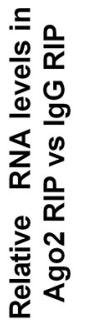

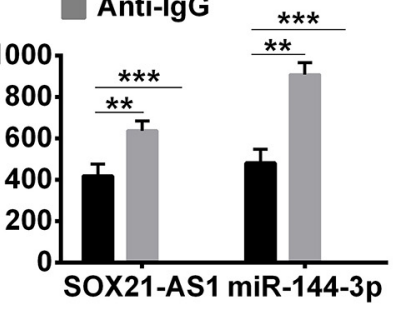

U87

Immunoprecipitation

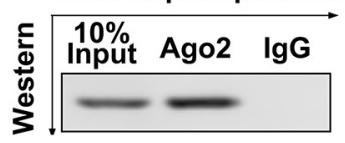

Input

Anti-Ago2

Anti-lgG

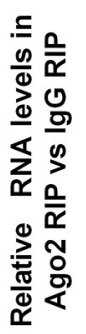

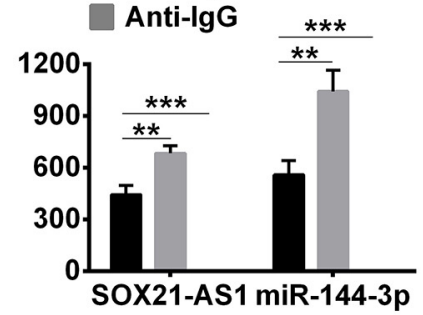

sh-NC

sh-SOX21-AS1

pcDNA3.1

pcDNA SOX21-AS1 pcDNA SOX21-AS1

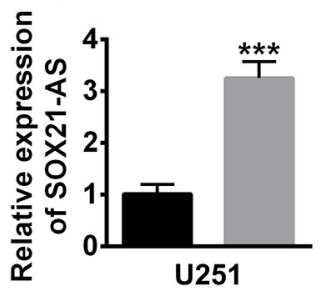

pcDNA SOX21-AS1

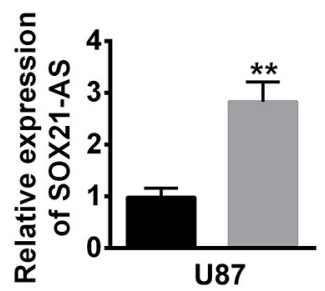

임
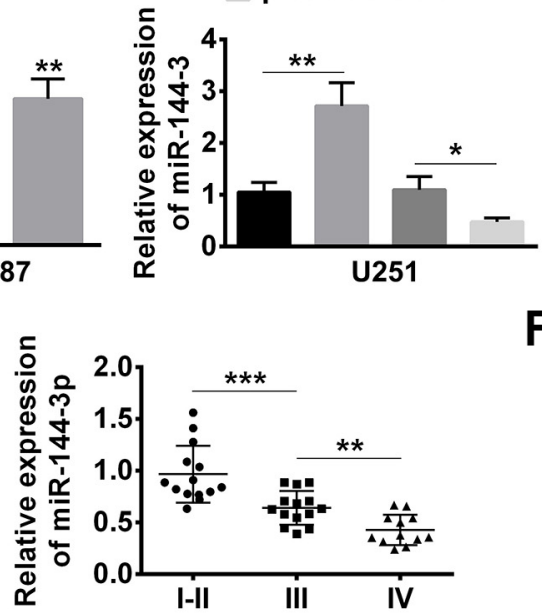

NC mimic

B.

miR-144-3p mimics
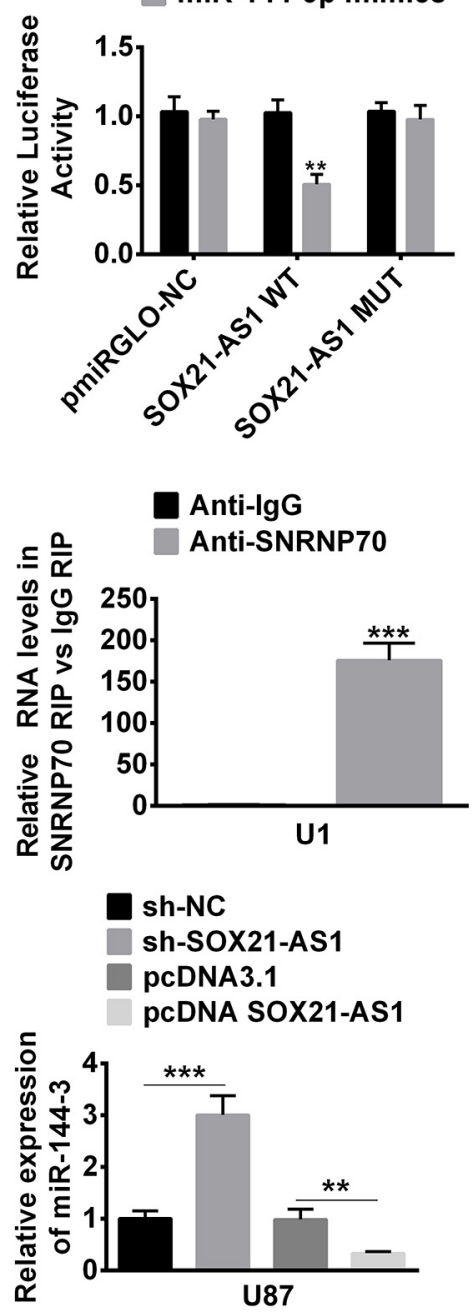

$\mathbf{F}$

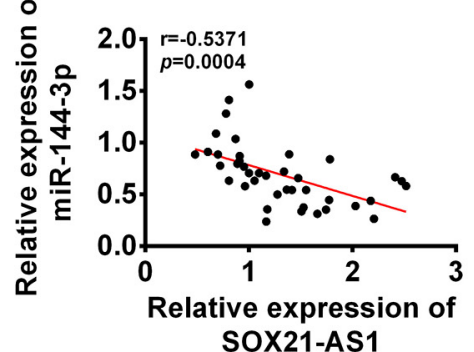

Figure 4. SOX21-AS1 was verified to be a target of miR-144-3. A) Bioinformatic analysis showed that SOX21-AS1 was a target of miR-144-3p. B and C) Interaction between SOX21-AS1 and miR-144-3 was determined via luciferase reporter assay and RIP assay. D and E) miR-144-3 level was analyzed via qRT-PCR. F) The negative correlation between SOX21-AS1 and miR-144-3 was showed. Data were presented as mean \pm SD. Statistical analysis was carried out using the student's test and one-way ANOVA by Tukey. ${ }^{* *} \mathrm{p}<0.01,{ }^{* * *} \mathrm{p}<0.001$.

Consistent with qRT-PCR detection, western blot analysis showed that miR-144-3p mimics decreased PAK7 level and miR-144-3p inhibitor increased PAK7 level in both U251 and U87 cells (Figure 5D). There was higher PAK7 expression in glioma tissues than normal tissues, in grade III gliomas than in grade I-II gliomas, in grade IV gliomas than grade III gliomas (Figure 5E). The negative correlation between miR-144-3p and PAK7, and positive correlation between SOX21-AS1 and PAK7 were shown (Figure 5F). These results indicated that miR-144-3p targeted PAK7. 
A

$\begin{array}{cr}\text { PAK7 WT } & \text { 5'-UCUUAACUAUUUCACAUACUGUA-3' } \\ \text { miR-144-3p } & \text { 3'-UCAUGUAGUAGAUAUGACAU-5' } \\ \text { PAK7 MUT } & \text { 5'-UCUUAACUAUUUCACUAUGACAA-3' }\end{array}$
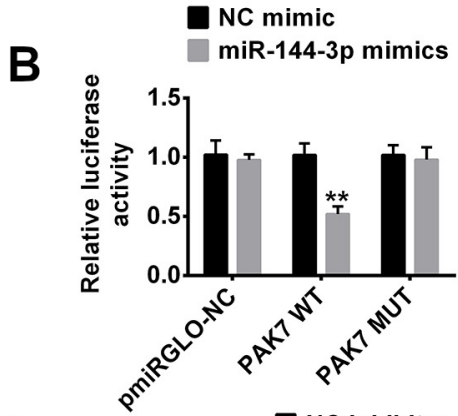

C

NC mimic miR-144-3p mimic
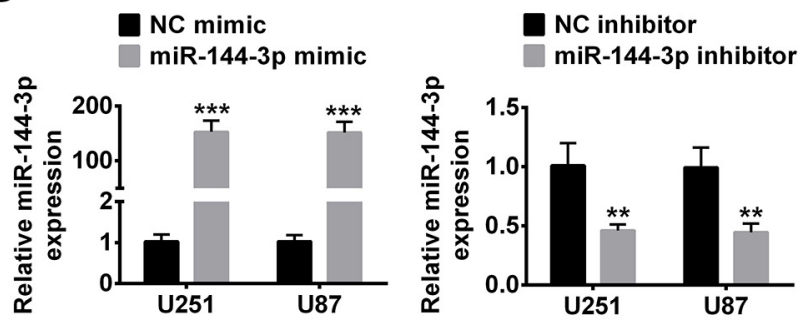

U251

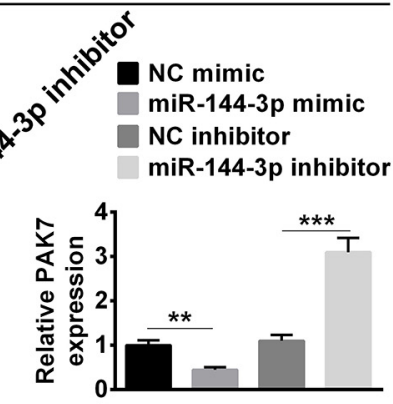

E
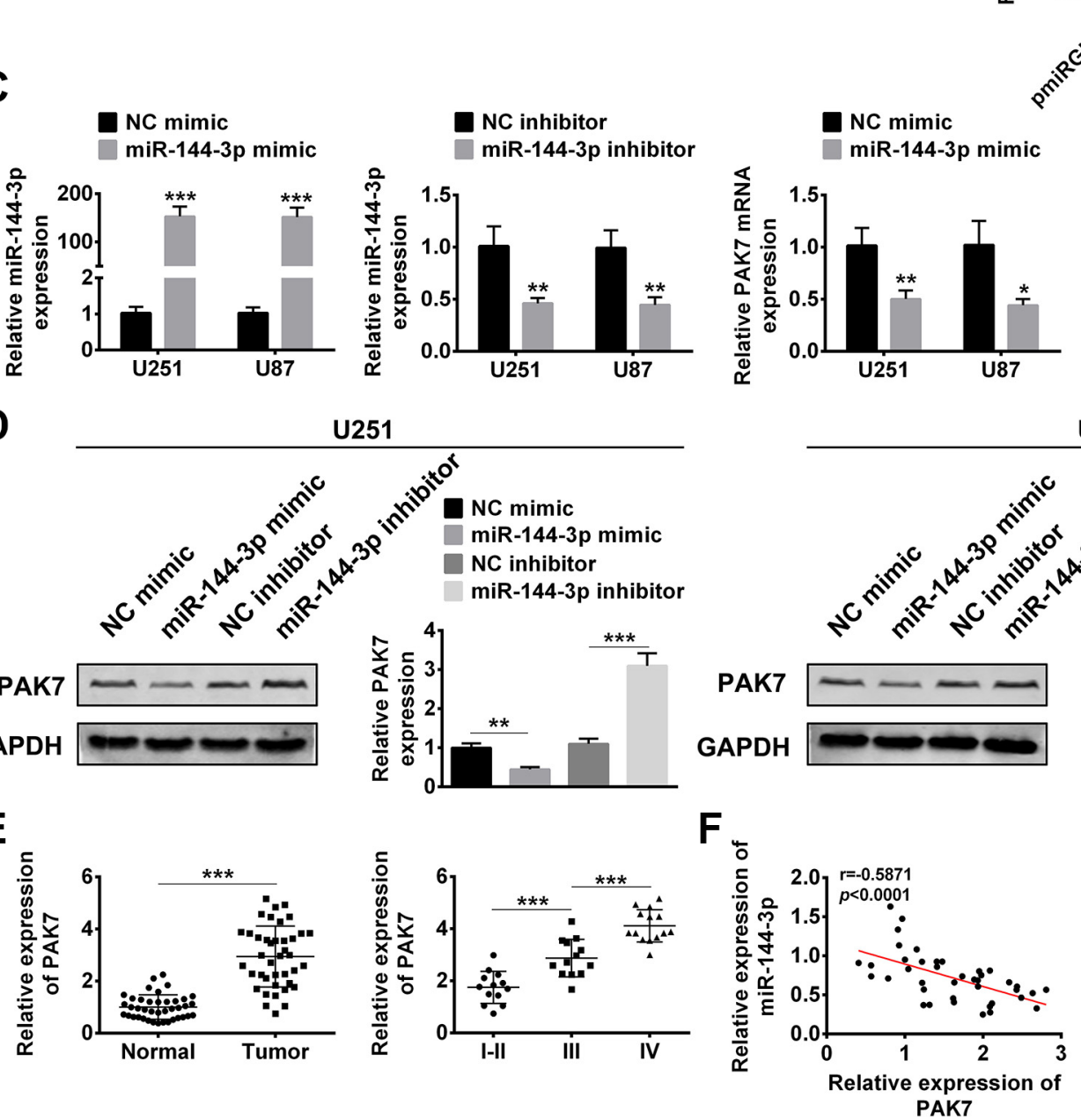

U87
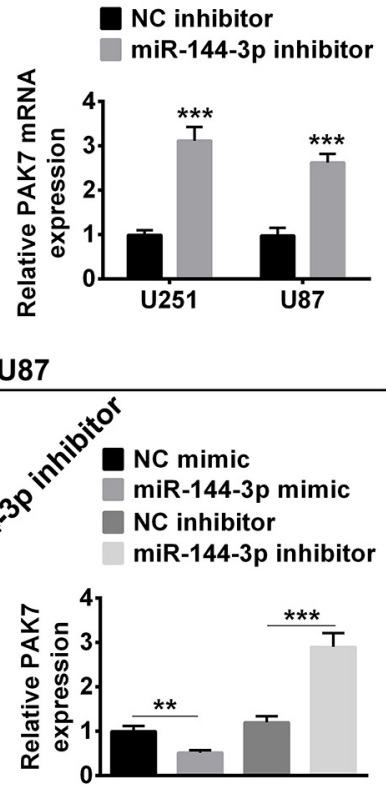

Figure 5. miR-144-3p targeted PAK7. A) Targetscan predicted that miR-144-3p targeted PAK7. B) Interaction between miR-144-3p and PAK7 was determined via luciferase reporter assay. C - E) PAK7 RNA and protein levels were detected through qRT-PCR and western blot analysis. F) The negative correlation between miR-144-3p and PAK7 and positive correlation between SOX21-AS1 and PAK7 were showed. Data were presented as mean \pm SD. Statistical analysis was carried out using the student's test and one-way ANOVA by Tukey. ${ }^{\star} p<0.05,{ }^{\star *} p<0.01,{ }^{* * *} p<0.001$.

SOX21-AS1, sponging miR-144-3p, affected proliferation and invasion in glioma. To further explore how SOX21-AS1 can affect proliferation and invasion in glioma by sponging miR-144-3p, the knockdown of SOX21-AS1 and miR-144-3p was performed in both U251 and U87 cells. CCK-8 assay showed that SOX21-AS1 knockdown inhibited proliferation and miR-144-3p knockdown promoted proliferation, whereas knockdown of both SOX21-AS1 and miR-144-3p elevated the proliferation induced by SOX21AS1 knockdown (Figure S1A). Moreover, SOX21-AS1 knockdown increased cell apoptosis, miR-144-3p knockdown decreased cell apoptosis, and knockdown of both SOX21-AS1 and miR-144-3p alleviated SOX21-AS1 knockdown-induced cell apoptosis (Figure S1B). Cell cycle detection revealed that the accumulation of cells at G1-stage and the reduction of cells at S-stage were caused by SOX21-AS1 knockdown, and the opposite effect on cell cycle was induced by miR-144-3p knockdown. Knockdown of both SOX21AS1 and miR-144-3p could alleviate SOX21-AS1 knockdown-induced accumulation of cells at G1-stage and reduc- 
tion of cells at S-stage (Figure S1C). Migration and invasion assay demonstrated that SOX21-AS1 knockdown showed a suppressive role in migration and invasion, and miR-144-3p knockdown enhanced migration and invasion. However, the migration and invasion induced by SOX21-AS1 knockdown were elevated in both U251 and U87 cells co-transfected with SOX21-AS1 knockdown and miR-144-3p knockdown (Figure S1D, S1E). These results suggested that SOX21-AS1, sponging miR-144-3p, affected proliferation and invasion in glioma.

SOX21-AS1, sponging miR-144-3p, affected proliferation and invasion by regulating $\mathrm{Wnt} / \boldsymbol{\beta}$-catenin pathway in glioma. To explore whether Wnt/ $\beta$-catenin pathway can be involved in the regulation of proliferation and invasion in glioma, western blot analysis was conducted. We found that SOX21-AS1 knockdown decreased PAK7. The important target genes for the Wnt canonical pathway, such as cyclin D1

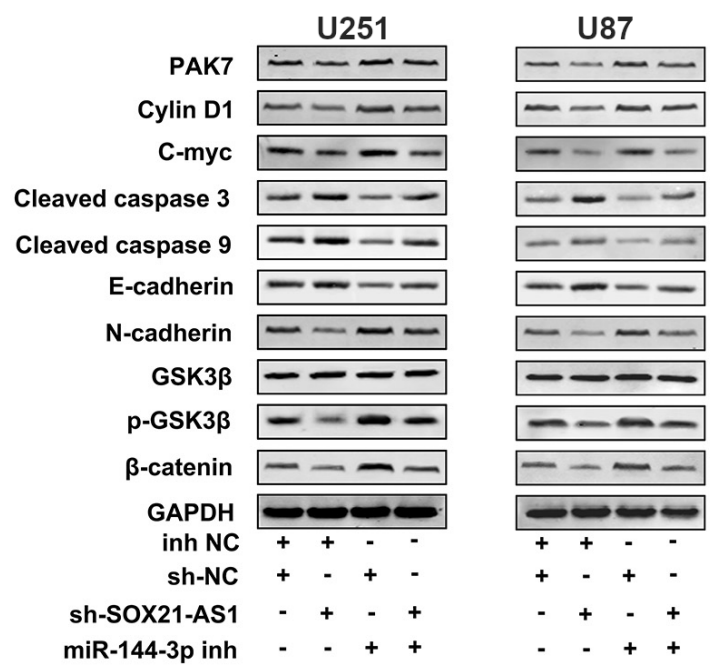

Figure 6. Western blot analysis examined the protein levels. Data were presented as mean \pm SD. Statistical analysis was carried out using oneway ANOVA by Tukey. ${ }^{*} \mathrm{p}<0.05,{ }^{* *} \mathrm{p}<0.01,{ }^{* *} \mathrm{p}<0.001$.

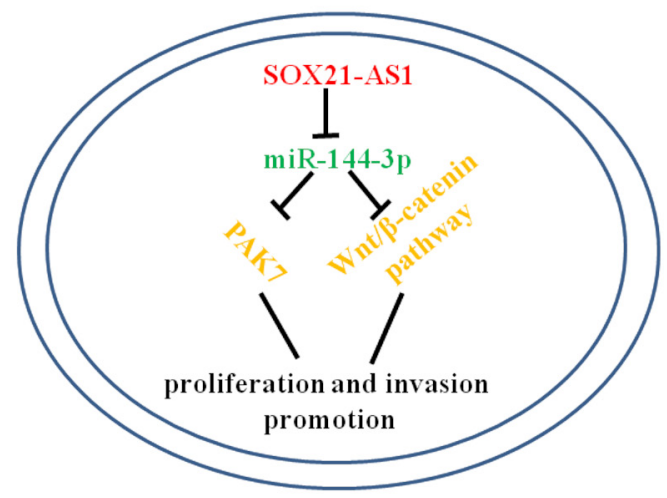

Figure 7. A schematic diagram that SOX21-AS1 promoted cell proliferation and invasion in glioma cells. and c-myc [37, 38], were suppressed by SOX21-AS1 knockdown. In addition, Wnt signaling affected the heterogeneous expression of epithelial-mesenchymal transition (EMT)associated factors, including $\mathrm{N}$-cadherin and E-cadherin [39]. N-cadherin was high in epithelial cells while E-cadherin was high in mesenchymal cells [39]. We demonstrated that SOX21-AS1 knockdown decreased N-cadherin protein level and increased E-cadherin protein level. $\beta$-catenin and GSK $3 \beta$, the key genes and proteins in Wnt/ $\beta$-catenin pathway [40], were measured. The results from western blot analysis proved that SOX21-AS1 knockdown repressed $\beta$-catenin and phosphorylated GSK3 $\beta$. Besides, both apoptosis-related proteins, cleaved caspase 3 and cleaved caspase 9 were elevated by SOX21-AS1 knockdown. Conversely, miR-144-3p knockdown showed the opposite effect on these protein levels. Moreover, these protein levels from glioma cells co-transfected with SOX21-AS1 and miR-144-3p knockdown were alleviated (Figure 6). These data indicated that SOX21-AS1, sponging miR-144-3p, affected proliferation and invasion by regulating $\mathrm{Wnt} / \beta$-catenin pathway in glioma.

\section{Discussion}

In this study, SOX21-AS1 level was upregulated in glioma tissues and cells. Then, SOX21-AS1 knockdown was conducted in glioma cells. Functional analysis revealed that SOX21-AS1 knockdown repressed cell proliferation and invasion in vitro. In addition, SOX21-AS1 knockdown inhibited tumor growth in vivo. We explored the interaction between SOX21-AS1 and miR-144-3p, and between miR-144-3p and PAK7. Moreover, a schematic diagram showed that SOX21-AS1/miR-144-3p/PAK7 showed an important role in regulating cell proliferation and invasion by Wnt/ $\beta$-catenin pathway in glioma (Figure 7 ).

Emerging evidence has shown that SOX21-AS1 could regulate the tumor progress. For example, SOX21-AS1 was high in hepatocellular carcinoma tissues, and SOX21-AS1 silencing was demonstrated to inhibit cell proliferation and metastasis [41]. High expression of SOX21-AS1 was reported to positively relate to tumor size in lung adenocarcinoma, and SOX21-AS1 knockdown was found to suppress proliferation, cell cycle as well as to cause cell apoptosis [22]. Like the previous studies, our study demonstrated that SOX21AS1 levels were dramatically increased in glioma tissues and cells. In addition, we also found that in vitro, SOX21-AS1 knockdown suppressed cell proliferation, reduced invasion and migration, and led to cell cycle arrest including accumulation of cells at G1-stage and reduction of cells at S-stage in both U251 and U87 cells. Moreover, cell apoptosis was elevated by SOX21-AS1 knockdown in these cells. In vivo, SOX21-AS1 knockdown reduced tumor tissue volume and weight. These results indicated that SOX21-AS1 knockdown showed a suppressive role in the progress of glioma.

Previously, in glioblastoma, low miR-144-3p expression was found in a glioblastoma cell line, and miR-144-3p 
was determined to inhibit tumor metastasis [27]. Interestingly, in our study, miR-144-3p was downregulated in glioma tissues. miR-144-3p knockdown promoted proliferation, migration, invasion, and inhibited cell apoptosis. It is reported that lncRNA may act as miRNA sponge to regulate gene expression [42]. Lu et al. demonstrated that LINC01503, via regulation of miR-4492/FOXK1, increased cell proliferation and invasion in colorectal cancer [43]. We et al. discovered that SOX21-AS1, targeting miR-145, was useful for tumorigenesis through myosin VI in colorectal cancer [23]. Accordingly, we verified that SOX21-AS1 could bind with miR-144-3p, and PAK7 was a target gene of miR-144-3p. Notably, PAK7 knockdown was reported to cause a reduction of cell migration and invasion in glioma [36]. In our study, the effects of SOX21-AS1 knockdowninduced proliferation, migration, invasion, and apoptosis were alleviated in glioma cells co-transfected with SOX21AS1 and miR-144-3p knockdown. Furthermore, in SOX21AS1 knockdown glioma cells, miR-144-3p knockdown attenuated SOX21-AS1 knockdown-induced decrease of PAK7, Cylin D1, C-myc, N-cadherin, p-GSK3 $\beta /$ GSK3 $\beta$ and $\beta$-catenin protein levels, increase of cleaved caspase 3 , cleaved caspase 9 and E-cadherin protein levels. Wang et al. proved that LINC01503 knockdown inhibited cell growth, invasion and activation of Wnt/ $\beta$-catenin pathway, decreasing $\beta$-catenin, cyclin D1 as well as c-myc levels [44]. These findings indicated that SOX21-AS1/miR-144-3p/ PAK7 axis played an oncogenic role in glioma cells by regulating Wnt/ $\beta$-catenin pathway.

In conclusion, we demonstrated that SOX21-AS1 was upregulated in glioma tissues and cells. SOX21-AS1 knockdown inhibited cell proliferation and invasion in vitro, and suppressed tumor growth in vivo. Moreover, our findings indicated that SOX21-AS1, sponging miR-144-3p, reduced cell proliferation and invasion in glioma via regulating PAK7 and $\mathrm{Wnt} / \beta$-catenin pathway. These results provide a new target for glioma therapy. However, more miRNAs may be sponged by SOX21-AS1. Further mechanisms of SOX21AS1 affecting development in glioma need to be explored. Additionally, the experiments about SOX21-AS1 applied into the clinical practice will be evaluated. Therefore, additional studies need to be performed in the future.

Supplementary information is available in the online version of the paper.

\section{References}

[1] JIANG Y, UHRBOM L. On the origin of glioma. Ups J Med Sci 2012; 117: 113-121. https://doi.org/10.3109/03009734.20 12.658976

[2] OSTROM QT, BAUCHET L, DAVIS FG, DELTOUR I, FISHER JL et al. The epidemiology of glioma in adults: a "state of the science" review. Neuro Oncol 2014; 16: 896-913. https://doi.org/10.1093/neuonc/nou087
[3] REES J, WATT H, JAGER HR, BENTON C, TOZER D et al. Volumes and growth rates of untreated adult low-grade gliomas indicate risk of early malignant transformation. Eur J Radiol 2009; 72: 54-64. https://doi.org/10.1016/j. ejrad.2008.06.013

[4] HASAN T, CARAGHER SP, SHIREMAN JM, PARK CH, ATASHI $\mathrm{F}$ et al. Interleukin-8/CXCR2 signaling regulates therapy-induced plasticity and enhances tumorigenicity in glioblastoma. Cell Death Dis 2019; 10: 292. https://doi. org/10.1038/s41419-019-1387-6

[5] CHEUNG NK, DYER MA. Neuroblastoma: developmental biology, cancer genomics and immunotherapy. Nat Rev Cancer 2013; 13: 397-411. https://doi.org/10.1038/nrc3526

[6] HAFAZALLA K, SAHGAL A, JAJA B, PERRY JR, DAS S. Procarbazine, CCNU and vincristine (PCV) versus temozolomide chemotherapy for patients with low-grade glioma: a systematic review. Oncotarget 2018; 9: 33623-33633. https://doi.org/10.18632/oncotarget.25890

[7] BUSH NA, CHANG SM, BERGER MS. Current and future strategies for treatment of glioma. Neurosurg Rev 2017; 40: 1-14. https://doi.org/10.1007/s10143-016-0709-8

[8] CHENG S, LU Y, XU S, LIU Q, LEE P. Cystoid angiocentric glioma: A case report and literature review. J Radiol Case Rep 2015; 9: 1-9. https://doi.org/10.3941/jrcr.v9i7.2413

[9] SUN J, SHI H, LAI N, LIAO K, ZHANG S et al. Overexpression of microRNA-155 predicts poor prognosis in glioma patients. Med Oncol 2014; 31: 911. https://doi.org/10.1007/ s12032-014-0911-x

[10] NIEDER C, ADAM M, MOLLS M, GROSU AL. Therapeutic options for recurrent high-grade glioma in adult patients: recent advances. Crit Rev Oncol Hematol 2006; 60: 181-193. https://doi.org/10.1016/j.critrevonc.2006.06.007

[11] FULLER GN. The WHO Classification of Tumours of the Central Nervous System, 4th edition. Arch Pathol Lab Med 2008; 132: 906. https://doi.org/10.1043/15432165(2008)132[906:TWCOTO]2.0.CO;2

[12] MERCER TR, DINGER ME, MATTICK JS. Long non-coding RNAs: insights into functions. Nat Rev Genet 2009; 10: 155-159. https://doi.org/10.1038/nrg2521

[13] OROM UA, DERRIEN T, BERINGER M, GUMIREDDY K, GARDINI A et al. Long noncoding RNAs with enhancer-like function in human cells. Cell 2010; 143: 46-58. https://doi. org/10.1016/j.cell.2010.09.001

[14] LIPOVICH L, JOHNSON R, LIN CY. MacroRNA underdogs in a microRNA world: evolutionary, regulatory, and biomedical significance of mammalian long non-protein-coding RNA. Biochim Biophys Acta 2010; 1799: 597-615. https:// doi.org/10.1016/j.bbagrm.2010.10.001

[15] FATIMA R, AKHADE VS, PAL D, RAO SM. Long noncoding RNAs in development and cancer: potential biomarkers and therapeutic targets. Mol Cell Ther 2015; 3: 5. https://doi. org/10.1186/s40591-015-0042-6

[16] GINGER MR, SHORE AN, CONTRERAS A, RIJNKELS M, MILLER J et al. A noncoding RNA is a potential marker of cell fate during mammary gland development. Proc Natl Acad Sci U S A 2006; 103: 5781-5786. https://doi. org/10.1073/pnas.0600745103 
[17] WANG P, XUE Y, HAN Y, LIN L, WU C et al. The STAT3binding long noncoding RNA lnc-DC controls human dendritic cell differentiation. Science 2014; 344: 310-313. https:// doi.org/10.1126/science.1251456

[18] LEUCCI E, VENDRAMIN R, SPINAZZI M, LAURETTE P, FIERS $\mathrm{M}$ et al. Melanoma addiction to the long non-coding RNA SAMMSON. Nature 2016; 531: 518-522. https://doi. org/10.1038/nature17161

[19] SUN M, KRAUS WL. From discovery to function: the expanding roles of long noncoding RNAs in physiology and disease. Endocr Rev 2015; 36: 25-64. https://doi.org/10.1210/ er.2014-1034

[20] GUPTA RA, SHAH N, WANG KC, KIM J, HORLINGS HM et al. Long non-coding RNA HOTAIR reprograms chromatin state to promote cancer metastasis. Nature 2010; 464: 1071-1076. https://doi.org/10.1038/nature08975

[21] KHAITAN D, DINGER ME, MAZAR J, CRAWFORD J, SMITH MA et al. The melanoma-upregulated long noncoding RNA SPRY4-IT1 modulates apoptosis and invasion. Cancer Res 2011; 71: 3852-3862. https://doi.org/10.1158/00085472.CAN-10-4460

[22] LU X, HUANG C, HE X, LIU X, JI J et al. A Novel Long Non-Coding RNA, SOX21-AS1, Indicates a Poor Prognosis and Promotes Lung Adenocarcinoma Proliferation. Cell Physiol Biochem 2017; 42: 1857-1869. https://doi. org/10.1159/000479543

[23] WEI AW, LI LF. Long non-coding RNA SOX21-AS1 sponges miR-145 to promote the tumorigenesis of colorectal cancer by targeting MYO6. Biomed Pharmacother 2017; 96: 953959. https://doi.org/10.1016/j.biopha.2017.11.145

[24] YANG CM, WANG TH, CHEN HC, LI SC, LEE MC et al. Aberrant DNA hypermethylation-silenced SOX21-AS1 gene expression and its clinical importance in oral cancer. Clin Epigenetics 2016; 8: 129. https://doi.org/10.1186/s13148016-0291-5

[25] MERCER TR, MATTICK JS. Structure and function of long noncoding RNAs in epigenetic regulation. Nat Struct Mol Biol 2013; 20: 300-307. https://doi.org/10.1038/nsmb.2480

[26] PAUL Y, THOMAS S, PATIL V, KUMAR N, MONDAL B et al. Genetic landscape of long noncoding RNA (lncRNAs) in glioblastoma: identification of complex lncRNA regulatory networks and clinically relevant lncRNAs in glioblastoma. Oncotarget 2018; 9: 29548-29564. https://doi.org/10.18632/ oncotarget. 25434

[27] CHENG ZX, SONG YX, WANG ZY, WANG Y, DONG Y. miR-144-3p serves as a tumor suppressor by targeting FZD7 and predicts the prognosis of human glioblastoma. Eur Rev Med Pharmacol Sci 2017; 21: 4079-4086.

[28] JAFFER ZM, CHERNOFF J. p21-activated kinases: three more join the Pak. Int J Biochem Cell Biol 2002; 34: 713-717. https://doi.org/10.1016/s1357-2725(01)00158-3

[29] ABO A, QU J, CAMMARANO MS, DAN C, FRITSCH A Et al. PAK4, a novel effector for Cdc42Hs, is implicated in the reorganization of the actin cytoskeleton and in the formation of filopodia. EMBO J 1998; 17: 6527-6540. https://doi. org/10.1093/emboj/17.22.6527
[30] YANG F, LI X, SHARMA M, ZARNEGAR M, LIM B et al Androgen receptor specifically interacts with a novel p21-activated kinase, PAK6. J Biol Chem 2001; 276: 15345-15353. https://doi.org/10.1074/jbc.M010311200

[31] DAN C, NATH N, LIBERTO M, MINDEN A. PAK5, a new brain-specific kinase, promotes neurite outgrowth in N1E-115 cells. Mol Cell Biol 2002; 22: 567-577. https://doi. org/10.1128/mcb.22.2.567-577.2002

[32] ZHU G, LI X, GUO B, KE Q, DONG M et al. PAK5-mediated $\mathrm{E} 47$ phosphorylation promotes epithelial-mesenchymal transition and metastasis of colon cancer. Oncogene 2016; 35: 1943-1954. https://doi.org/10.1038/onc.2015.259

[33] LI D, YAO X, ZHANG P. The overexpression of P21-activated kinase 5 (PAK5) promotes paclitaxel-chemoresistance of epithelial ovarian cancer. Mol Cell Biochem 2013; 383: 191199. https://doi.org/10.1007/s11010-013-1767-7

[34] ABURATANI T, INOKUCHI M, TAKAGI Y, ISHIKAWA T, OKUNO K et al. High expression of P21-activated kinase 5 protein is associated with poor survival in gastric cancer. Oncol Lett 2017; 14: 404-410. https://doi.org/10.3892/ ol.2017.6115

[35] GU X, WANG C, WANG X, MA G, LI Y et al. Efficient inhibition of human glioma development by RNA interferencemediated silencing of PAK5. Int J Biol Sci 2015; 11: 230-237. https://doi.org/10.7150/ijbs.9193

[36] HAN ZX, WANG XX, ZHANG SN, WU JX, QIAN HY et al. Downregulation of PAK5 inhibits glioma cell migration and invasion potentially through the PAK5-Egr1-MMP2 signaling pathway. Brain Tumor Pathol 2014; 31: 234-241. https:// doi.org/10.1007/s10014-013-0161-1

[37] HE TC, SPARKS AB, RAGO C, HERMEKING H, ZAWEL L et al. Identification of c-MYC as a target of the APC pathway. Science 1998; 281: 1509-1512. https://doi.org/10.1126/science.281.5382.1509

[38] TETSU O, MCCORMICK F. Beta-catenin regulates expression of cyclin D1 in colon carcinoma cells. Nature 1999; 398: 422-426. https://doi.org/10.1038/18884

[39] Liu CC, Cai DL, Sun F, Wu ZH, Yue B et al. FERMT1 mediates epithelial-mesenchymal transition to promote colon cancer metastasis via modulation of beta-catenin transcriptional activity. Oncogene 2017; 36: 1779-1792. https://doi. org/10.1038/onc.2016.339

[40] ZHOU J, LING J, SONG H, LV B, WANG L et al. Neurokinin-1 receptor is a novel positive regulator of Wnt/ betacatenin signaling in melanogenesis. Oncotarget 2016; 7: 81268-81280. https://doi.org/10.18632/oncotarget.13222

[41] WEI C, WANG H, XU F, LIU Z, JIANG R. LncRNA SOX21AS1 is associated with progression of hepatocellular carcinoma and predicts prognosis through epigenetically silencing p21. Biomed Pharmacother 2018; 104: 137-144. https://doi. org/10.1016/j.biopha.2018.05.010

[42] SHAN Z, AN N, QIN J, YANG J, SUN H et al. Long noncoding RNA Linc00675 suppresses cell proliferation and metastasis in colorectal cancer via acting on miR-942 and Wnt/ beta-catenin signaling. Biomed Pharmacother 2018; 101: 769-776. https://doi.org/10.1016/j.biopha.2018.02.123 
[43] LU SR, LI Q, LU JL, LIU C, XU X et al. Long non-coding RNA LINC01503 promotes colorectal cancer cell proliferation and invasion by regulating miR-4492/FOXK1 signaling. Exp Ther Med 2018; 16: 4879-4885. https://doi.org/10.3892/ etm.2018.6775
[44] WANG H, SHENG ZG, DAI LZ. Long non-coding RNA LINC01503 predicts worse prognosis in glioma and promotes tumorigenesis and progression through activation of Wnt/ beta-catenin signaling. Eur Rev Med Pharmacol Sci 2019; 23: 1600-1609. https://doi.org/10.26355/eurrev_201902_17119 


\section{Long non-coding RNA SOX21-AS1 promotes cell proliferation and invasion through upregulating PAK7 expression by sponging miR-144-3p in glioma cells}

S. Y. GAI ${ }^{1}$, Z. H. YUAN ${ }^{2, *}$

Supplementary Information

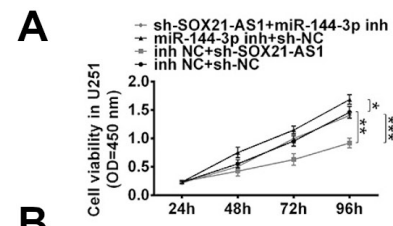

B.
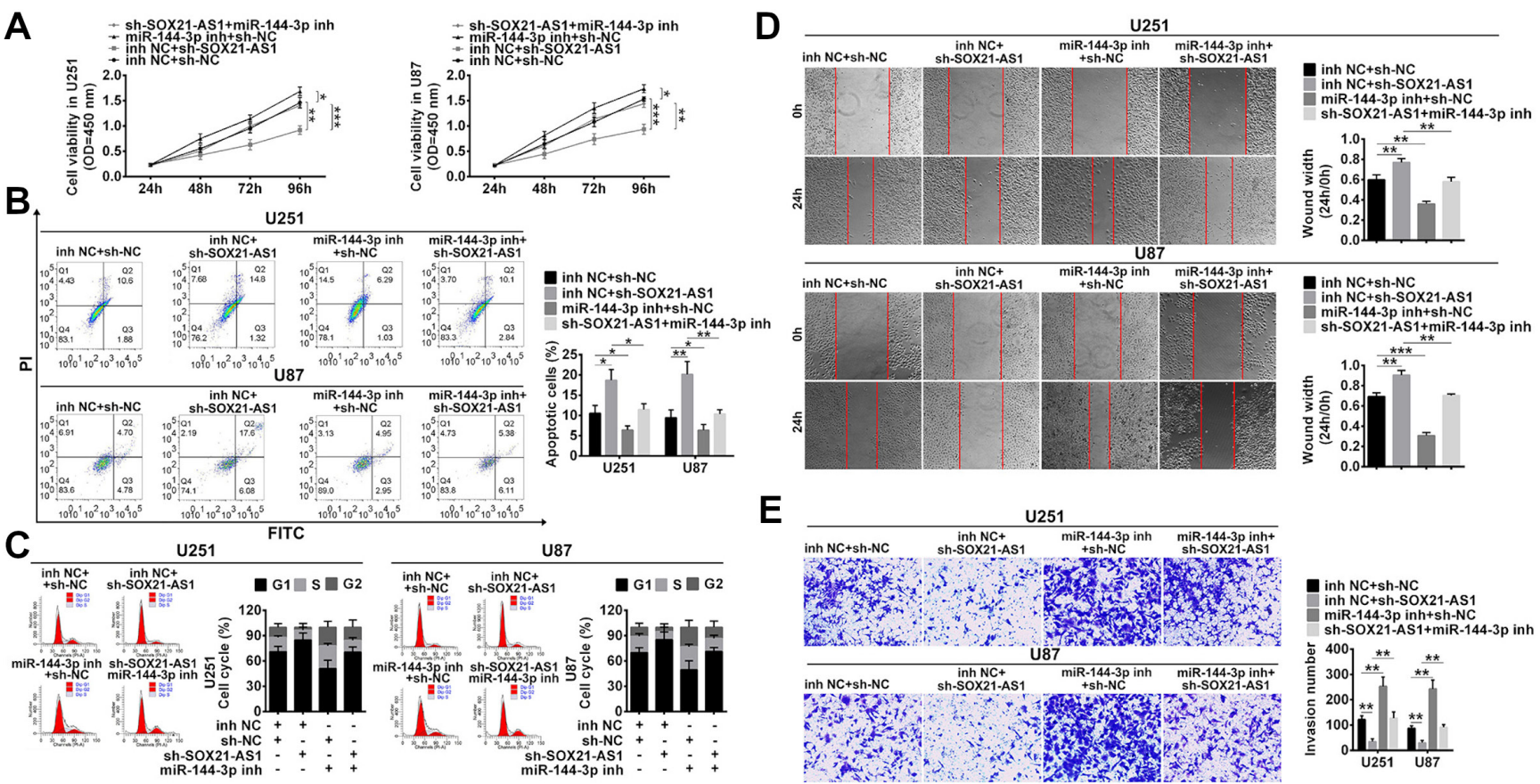

E
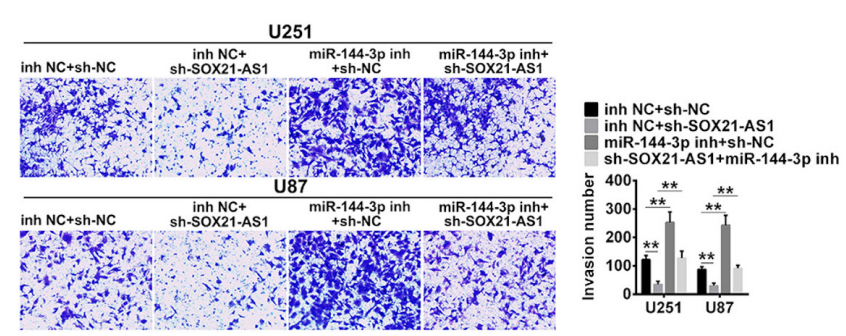

Figure S1. SOX21-AS1, sponging miR-144-3p, affected proliferation and invasion by regulating Wnt/ $\beta$-catenin pathway in glioma. A) CCK-8 assay detected cell proliferation. B and C) Cell apoptosis and cell cycle detection was conducted using flow cytometry was used for the analysis. D and E) Cell migration and invasion was measured via scratch wound healing assay and transwell assay. Data were presented as mean \pm SD. Statistical analysis was carried out using one-way and two-way ANOVA by Tukey. ${ }^{*} \mathrm{p}<0.05,{ }^{* *} \mathrm{p}<0.01,{ }^{* * *} \mathrm{p}<0.001$. 